\title{
Brain Developmental Differences Between Preterm-born Twins and Singletons: A Multi-modal MRI Study
}

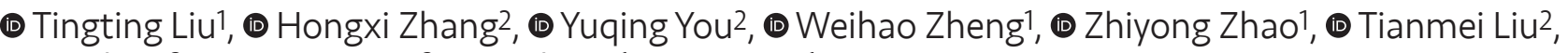 \\ (1) Xiaoli Su2, (1) Fengyu Tian22, (1) Yi Zhang1, (1) Dan Wu¹
}

${ }^{1}$ Key Laboratory for Biomedical Engineering of Ministry of Education, Department of Biomedical Engineering, College of Biomedical Engineering \& Instrument Science, Zhejiang University, Hangzhou, Zhejiang, China

2Zhejiang University Faculty of Medicine, Children's Hospital, Clinic of Radiology, Hangzhou, China

\begin{abstract}
Aim: Twin studies allow for the investigation of genetic and environmental influences on human brain development. The generalizability of their findings depends on the developmental similarity between twins and singletons. This study aimed to evaluate the structural and functional differences in a cohort of preterm-born twins and singletons at term-equivalent age.

Materials and Methods: Eighteen twins and forty-seven singletons were included and scanned at the term-equivalent age. Brain volumes from 3D T1-weighted images, quantitative metrics and structural connectivity from diffusion tensor imaging, and low-frequency brain activity and functional connectivity from resting-state functional MRI ( $r$-fMRI) were obtained from these neonates.

Results: We found no significant volumetric differences after multiple comparison correction. The diffusivity values in the cingulum cingular part, cingulate gyrus, lateral fronto-orbital gyrus, gyrus rectus, as well as medial fronto-orbital gyrus were significantly higher in the twin group than in the singleton group. Structural connectivity analysis showed higher transitivity in the twin group compared to the singletons, indicating increased local connectivity. For rs-fMRI, the twin group showed greater fractional amplitude of low-frequency fluctuation (fALFF) values in the salience network and several fronto-temporal regions compared with the singleton group. It is worth noting that we found differences both in structural and functional measurements (MD and fALFF) in the prefrontal and cingulate cortex.

Conclusion: The structural and functional differences collectively indicated that preterm-born twins may have delayed brain development compared with gestational age-matched singletons at term-equivalent age, which may be related to perinatal-neonatal problems.
\end{abstract}

Keywords: Twin-singleton, brain development, preterm-birth, multi-modal MRI, connectivity

\section{Introduction}

Knowledge from twin studies allows researchers to understand the contribution of genetic and environmental factors to brain development (1-5). The generalizability of these studies, however, depends on the assumption that brain developmental patterns in twins are comparable to those in singletons. It has been well established that twins have compromised growth in the third trimester starting from about 30 weeks of gestation that may be attributed to certain reasons, such as the different growth

Address for Correspondence

Dan Wu, Key Laboratory for Biomedical Engineering of Ministry of Education, Department of Biomedical Engineering, College of Biomedical Engineering \& Instrument Science, Zhejiang University, Hangzhou, Zhejiang, China

Phone: +86-15825508242 E-mail: danwu.bme@zju.edu.cn ORCID: orcid.org/0000-0002-9303-5821

Received: 09.11.2020 Accepted: 22.02.2021

${ }^{\circ}$ Copyright 2021 by Ege University Faculty of Medicine, Department of Pediatrics and Ege Children's Foundation The Journal of Pediatric Research, published by Galenos Publishing House. 
pattern, placental size, maternal nutrition, and family care after birth (6-8), leading to potential brain developmental differences between twins and singletons.

Neuroimaging, especially magnetic resonance imaging (MRI), has been shown to be a powerful tool to characterize the structural and functional changes in the developing brain. Brain MRI has been employed to investigate twins and singletons, but not to its full potential. Several existing studies (9-11) focused on morphological differences between twin and singleton brains from 10 months to 30 years of age, however, their conclusions were inconsistent. A recent longitudinal study by Sadeghi et al. (12) compared longitudinal diffusion tensor imaging (DTI) metrics between singletons and twins from birth to 2 years old, and showed that the axial diffusivities in the anterior limb of the internal capsule and anterior corona radiata were significantly higher in twins compared with singletons during early development.

Current MRI studies comparing twins and singletons are still limited and their findings are discrepant, and most studies used a single MRI modality (T1-weighted or DTI). Moreover, all the aforementioned studies focused on comparing twins with term-born singletons, while it is known that twins have an increased risk of preterm delivery (13). The unpaired gestational age makes it difficult to separate the effects of twin birth from premature birth in the observed developmental differences compared with singletons (14). Here, we aim to systematically evaluate whether the MRI findings of twins can be generalized to singleton studies by comparing the preterm-born singleton and twin neonates that were born with equivalent gestational ages with a multi-modal MRI approach, including morphological MRI, DTI, and resting-state functional MRI (rs-fMRI) acquired at term-equivalent age.

\section{Materials and Methods}

\section{Subjects}

Preterm-born infants were enrolled at term-equivalent age for MRI scan. Ethical approval was obtained from the Institutional Review Board at the Children's Hospital of Zhejiang University School of Medicine (2019-11-13). Written informed consent was provided by the parents. Exclusion criteria included 1) congenital malformation or syndrome; 2) acquired brain injury on MRI; 3) visible artifacts on MRI, or a mean frame-wise displacement (FD) exceeding $0.2 \mathrm{~mm}$ for rs-fMRI; 4) psychiatric or neurological family history;
5) pregnancy complications; 6) illicit drug or alcohol use during pregnancy.

\section{Image Acquisition}

All neonates received $50 \mathrm{mg} / \mathrm{kg}$ oral or enema chloral hydrate 30 minutes before scanning from a radiology nurse who was trained and certified to administer sedation. Ear protectors and physiological monitors were used for protection and monitoring. The scans were performed on a Siemens 1.5T Avanto MRI scanner (Siemens Healthcare, Erlangen, Germany) with a 12-channel Siemens head coil. The multi-modal MRI protocol included the following: 1) 3D sagittal T1-weighted imaging using the MPRAGE sequence with repetition time $(T R)=1,910 \mathrm{~ms}$, echo time $(T E)=3.01 \mathrm{~ms}$, inversion time $=1,100 \mathrm{~ms}$, flip angle $=15^{\circ}$, resolution $=0.82 \times 0.82 \times 1$ $\mathrm{mm}^{3}$, field of view $(F O V)=210 \times 210 \times 160 \mathrm{~mm}^{3}$, and acquisition matrix $=256 \times 256 \times 160$; 2) DTI using a single-shot echoplanar imaging (EPI) sequence with $\mathrm{TR}=3,800 \mathrm{~ms}, \mathrm{TE}=86$ $\mathrm{ms}$, in-plane resolution $=1.64 \times 1.64 \mathrm{~mm}^{2}, F O V=210 \times 210$ $\mathrm{mm}^{2}, 25$ slices at a slice thickness of $5 \mathrm{~mm}, 12$ gradient directions at a b-value of $750 \mathrm{~s} / \mathrm{mm}^{2}$ and 1 non-weighted image (b0), and 4 repetitions; 3 ) rs-fMRI using a gradientecho $\mathrm{T}^{*}$-weighted $\mathrm{EPI}$ sequence with $\mathrm{TR}=2,000 \mathrm{~ms}, \mathrm{TE}=40$ $\mathrm{ms}$, in-plane resolution $=3.28 \times 3.28 \mathrm{~mm}^{2}, F O V=210 \times 210$ $\mathrm{mm}^{2}, 24$ slices at a slice thickness of $6 \mathrm{~mm}$, bandwidth $=200$ $\mathrm{Hz} /$ pixel, and number of volumes $=180$.

\section{Image Processing}

\section{Image segmentation and structural volumes}

The 3D T1-weighted images went through a fully automated segmentation pipeline via the online platform MRICloud (www.mricloud.org) (15), which performed a multi-atlas based segmentation, based on the JHU neonatal multi-atlas (16). Thirty-eight regions of interest (ROIs) were defined, including the gray matter, myelinated/ unmyelinated white matter, brain stem, corpus callosum, caudate, putamen, thalamus, and cerebrospinal fluid (CSF), etc. The volumes of the ROls were obtained and then summed over the two hemispheres (resulting in 18 symmetric ROls after removing two non-brain-tissue ROIs), assuming negligible laterality in this study.

\section{DTI processing and structural connectivity}

\section{a) Pre-processing and tensor reconstruction}

All data were manually inspected by a radiologist (T.L.) to exclude diffusion-weighted images (DWI) with 
noticeable imaging artifacts, followed by intra-subject registration using a 12-parameter affine transformation (17) to correct for head motion. Then, we employed the standard preprocessing steps with denoising (18), Gibb's ringing removal (19), distortion correction (20), and bias field correction (21). Fractional anisotropy (FA), mean/axial/ radial diffusivity (MD/AD/RD) maps were generated from the diffusion tensor using the weighted linear least squares method.

\section{b) Segmentation}

The individual DTI data were transformed to the JHUneonate single brain DTI atlas for image segmentation (22). The individual mean DWI images were aligned to the atlas DWI image with an affine transformation, followed by histogram matching between the atlas and subject images. Following this, the non-linear transformation was performed with large deformation diffeomorphic metric mapping $(23,24)$, utilizing multi-channel contrasts of the mean DWI, FA, and b0 images (25). The DTI images were further inspected for registration failure, and none of the data showed visible registration errors. After transforming to the atlas space, the individual images were automatically segmented into $126 \mathrm{ROIs}$, as defined in the JHU-neonate atlas. The $F A, M D, A D$, and $R D$ values were extracted from the ROls, and an MD threshold of $2 \times 10-3 \mathrm{~mm}^{2} / \mathrm{s}$ was used to exclude the CSF voxels. The DTI metrics were then averaged over the hemispheres for statistical analysis.

\section{c) Tractography and structural connectivity}

Tractography was performed on the pre-processed DWIs by a tensor-based probabilistic fiber tracking algorithm (26) in MRtrix3 (www.mrtrix.org). The seed voxels were selected randomly within a whole-brain mask, and the following tracking parameters were used: cut-off of 0.06, step size of $0.16 \mathrm{~mm}$, minimum/maximum length of $8 / 164 \mathrm{~mm}$. Probabilistic tractography was used in this study as it was shown to yield higher connectome reproducibility than the deterministic method $(27,28)$.

To construct the structural network, all deep white matter and cerebellum ROIs were excluded, leaving 62 ROIs as structural network nodes. It is worth noting that the network nodes included not only cortical and subcortical GM but also subcortical WM because the subcortical WM helped to determine the fibers linked to cortical regions (29). The weakest $1 \%$ of the connections, which were considered as spurious streamlines, were discarded. Following this, the streamlines were log-transformed to achieve normality (30). Seven network parameters were calculated using the brain connectivity toolbox (31), including the degree, transitivity, local efficiency, global efficiency, modularity, characteristic path length, and small-worldness.

\section{rs-fMRI Processing}

\section{a) Pre-processing}

The rs-fMRI data were preprocessed using the data processing assistant for resting-state fMRI (DPARSF, Advanced Edition) (32). First, the first ten time points were removed, followed by slice-timing correction and head motion correction, and those subjects with mean FD exceeding $0.2 \mathrm{~mm}$ were excluded (33). Following this, spatial normalization was performed via T1-weighted anatomical images that were registered to the JHU-neonate single brain T1 atlas (22), and all fMRI images were resampled to $3 \mathrm{~mm}$ isotropic voxels using SPM8. Next, the normalized images were smoothed with the Full Width at Half Maximum set at $6 \mathrm{~mm}$. Finally, linear drift was removed, and the six rigid head motion parameters, as well as sources of physiological artifact extracted from white matter and CSF masks, were regressed out. In addition, bad time points were scrubbed using a threshold of Jenkinson FD>0.2 mm as well as one volume before and two volumes after (33).

\section{b) ALFF, fALFF and functional connectivity}

The low-frequency fluctuations were quantified by the amplitude of low-frequency fluctuation (ALFF) and fractional ALFF (fALFF) (34). ALFF was calculated within a specific low-frequency range $(0.01-0.1 \mathrm{~Hz})$, then the ratio of the power of the low-frequency band to that of the entire frequency range was calculated as fALFF. Z-transform was performed on both ALLF and fALFF to improve the normality before filtering. Then, the functional connectivity was calculated based on ALFF or fALFF, using the modified atlas as mentioned in 2.3.2 (c), and the correlation coefficient matrices were converted into $z$ map by Fisher's r-to-z transform to improve normality. Correlation coefficients under 0.2 , which were considered to be a negligible correlation, were discarded (35). The same network parameters were calculated as those in the structural network.

\section{Statistical Analysis}

For demographic information, categorical data were analyzed using the chi-square test. Shapiro-Wilk's test was used to analyze the distribution of measurement data. The Student's t-test was used for normally distributed data, and the Mann-Whitney $U$ test applied to data that did not fulfill the requirements for normality. For the differences in DTI 
and network metrics between twins and singletons, analysis of covariance (ANCOVA) was performed with a permutation approach in R-Project $(36,37)$. Then the p-values of DTI metrics extracted from multiple ROls were adjusted with the Bonferroni method. For ALFF and fALFF, a voxel-based analysis of differences between groups was performed with DPABI (32) using a permutation test method followed by Bonferroni correction for multiple testing using thresholdfree cluster enhancement (38). The significance level was set at 0.05 for all tests. For all analyses, gender, birth weight, postmenstrual age (PMA) at scan, and Apgar score at 5 minutes after birth were used as covariates.

\section{Results}

\section{Demographic and clinical characteristics}

In total, eighteen twins and forty-seven singletons were included in this study. For rs-fMRI analysis, eight singletons were excluded as their mean FD exceeded 0.2

Table I. Demographic and basic clinical information characteristics of study participants

\begin{tabular}{|l|l|l|l|}
\hline & Twins & Singletons & p-value \\
\hline Gender (male/female) & $12 / 6$ & $23 / 24$ & $0.315^{\mathrm{a}}$ \\
\hline PMA at birth (weeks) & $32.06 \pm 1.00$ & $32.13 \pm 0.99$ & $0.796^{\mathrm{b}}$ \\
\hline PMA at scan (weeks) & $40.50 \pm 0.99$ & $40.06 \pm 1.55$ & $0.185^{\mathrm{b}}$ \\
\hline Birth weight (grams) & $1,802.5 \pm 254.1$ & $1,850.6 \pm 381.0$ & $0.559^{\mathrm{b}}$ \\
\hline $\begin{array}{l}\text { Apgar score at 5-min } \\
\text { after birth }\end{array}$ & $9.83 \pm 0.38$ & $9.74 \pm 0.53$ & $0.460^{\mathrm{b}}$ \\
\hline $\begin{array}{l}\text { Data are represented as mean } \pm \text { standard deviation. } \\
\text { aCompared by chi-square test, }{ }^{\mathrm{b} C o m p a r e d ~ b y ~ M a n n-W h i t n e y ~ U ~ t e s t ~}\end{array}$ \\
\hline
\end{tabular}

Supplementary Table I. Demographic and basic clinical characteristics of study participants used in rs-fMRI analysis after removing eight singletons with significant head movement (FD>0.2 mm)

\begin{tabular}{|l|l|l|l|}
\hline & Twin & Singleton & p-value \\
\hline $\begin{array}{l}\text { Gender (male/ } \\
\text { female) }\end{array}$ & $12 / 6$ & $18 / 21$ & $0.168^{\mathrm{a}}$ \\
\hline PMA at birth/weeks & $32.06 \pm 1.00$ & $32.21 \pm 0.73$ & $0.575^{\mathrm{b}}$ \\
\hline PMA at scan/week & $40.50 \pm 0.99$ & $40.15 \pm 1.62$ & $0.324^{\mathrm{b}}$ \\
\hline Birth weight/gram & $1802.5 \pm 254.1$ & $1835.8 \pm 364.8$ & $0.692^{\mathrm{b}}$ \\
\hline $\begin{array}{l}\text { Apgar score at 5 min } \\
\text { after birth }\end{array}$ & $9.83 \pm 0.38$ & $9.74 \pm 0.55$ & $0.480^{\mathrm{b}}$ \\
\hline $\begin{array}{l}\text { Data was represented as mean } \pm \text { standard deviation } \\
\text { PMA: Postmenstrual age, FD: Frame-wise displacement, rs-fMRI: Resting-state functional } \\
\text { magnetic resonance imaging }\end{array}$
\end{tabular}

$\mathrm{mm}$. Demographic and basic clinical information of the 65 study participants is provided in Table I, and the information of the 57 subjects used for rs-fMRI analysis is listed in Supplementary Table I. No significant group difference was found in terms of the listed demographic and clinical characteristics.

\section{Comparison of the structural volumes}

Based on the automated segmentation of the 3D T1-weighted images, the structural volumes of the $18 \mathrm{ROIs}$ were compared between the twins and singletons. The myelinated white matter and thalamus in the singleton group demonstrated higher volumes than the twin group ( $p=0.034$ and $p=0.012$, respectively before correction), but no significant difference was found after multiple comparison correction. The structural volumes and the statistical test results are listed in Supplementary Table II.

\section{Comparison of the DTI metrics}

As demonstrated in Figure 1, the MD values of the cingulum cingular part (CGC), cingulate gyrus (CingG), lateral fronto-orbital gyrus (LFOG), gyrus rectus (RG), as well as medial fronto-orbital gyrus in the twin group were significantly higher than the singleton group (adjusted $p<0.05)$. The $A D$ and $R D$ were also increased in these regions (Figure 1), while RD showed differences in three additional ROls, including the lingual gyrus, fusiform gyrus (Fu), and cingulum hippocampal part. The color maps in Figure 2 demonstrate the spatial distribution of regions with significant differences in MD between the groups, and the colors indicated the percentage of group difference by

Table II. Brain regions that show significant differences in fALFF between the twin and singleton groups

\begin{tabular}{|c|c|c|c|c|c|}
\hline \multirow{2}{*}{ Brain region } & \multirow{2}{*}{$\begin{array}{l}\text { Cluster size } \\
\text { (number of } \\
\text { voxels) }\end{array}$} & \multirow{2}{*}{$\begin{array}{l}\mathbf{t} \\
\text { values }\end{array}$} & \multicolumn{3}{|c|}{ MNI (peak) } \\
\hline & & & $x$ & y & z \\
\hline Ins L & \multirow{4}{*}{147} & \multirow{4}{*}{4,025} & \multirow{4}{*}{-27} & \multirow{4}{*}{-4} & \multirow{4}{*}{4} \\
\hline STG L & & & & & \\
\hline LFOG L & & & & & \\
\hline PoCG L & & & & & \\
\hline STG R & \multirow{3}{*}{101} & \multirow{3}{*}{4,268} & \multirow{3}{*}{21} & \multirow{3}{*}{14} & \multirow{3}{*}{-17} \\
\hline Ins R & & & & & \\
\hline LFOG R & & & & & \\
\hline CingG & \multirow{2}{*}{64} & \multirow{2}{*}{4,123} & \multirow{2}{*}{0} & \multirow{2}{*}{17} & \multirow{2}{*}{-14} \\
\hline RG & & & & & \\
\hline $\begin{array}{l}\text { Ins: Insular corte } \\
\text { LFOG: Lateral fro } \\
\text { gyrus, fALFF: Fra }\end{array}$ & $\begin{array}{l}\text { CingG: Cingula } \\
\text {-orbital gyrus, } \\
\text { mal amplitude }\end{array}$ & $\begin{array}{l}\text { rtex, STG: } \\
\text { yrus rectus } \\
\text { l-frequenc }\end{array}$ & $\begin{array}{l}\text { Supe } \\
\text { POC } \\
\text { fluc }\end{array}$ & $\begin{array}{l}\text { temp } \\
\text { Left }\end{array}$ & $\begin{array}{l}\text { gyrus } \\
\text { entra }\end{array}$ \\
\hline
\end{tabular}


Supplementary Table II. Statistical results of structural volume differences between twins and singletons

\begin{tabular}{|l|l|l|}
\hline ROIs & p-values & Adjusted p-values \\
\hline Intracranial volume & 0.586 & 1 \\
\hline CSF & 0.836 & 1 \\
\hline Lateral ventricle & 0.646 & 1 \\
\hline $3^{\text {rd }}$ ventricle & 0.395 & 1 \\
\hline $4^{\text {th }}$ Ventricle & 0.455 & 1 \\
\hline $\begin{array}{l}\text { Cavum septum } \\
\text { pellucidum }\end{array}$ & 0.859 & 1 \\
\hline Gray matter & 0.671 & 1 \\
\hline White matter & 0.388 & 1 \\
\hline $\begin{array}{l}\text { Myelinated white } \\
\text { matter }\end{array}$ & $\mathbf{0 . 0 3 4}$ & 0.605 \\
\hline Brain stem & 0.114 & 1 \\
\hline Cerebellum & 0.728 & 1 \\
\hline Corpus callosum & 0.210 & 1 \\
\hline Caudate & 0.999 & 1 \\
\hline Putamen & 0.818 & 1 \\
\hline Globus pallidus & 0.837 & 1 \\
\hline Thalamus & $\mathbf{0 . 0 1 2}$ & 0.223 \\
\hline Hippocampus & 0.967 & 1 \\
\hline Amygdala & 0.413 & 1 \\
\hline $\begin{array}{l}\text { P-values before and after the Bonferroni correction were shown. } \\
\text { ROI: Region of interest, CSF: Cerebrospinal fluid }\end{array}$ & & \\
\hline & & \\
\hline
\end{tabular}

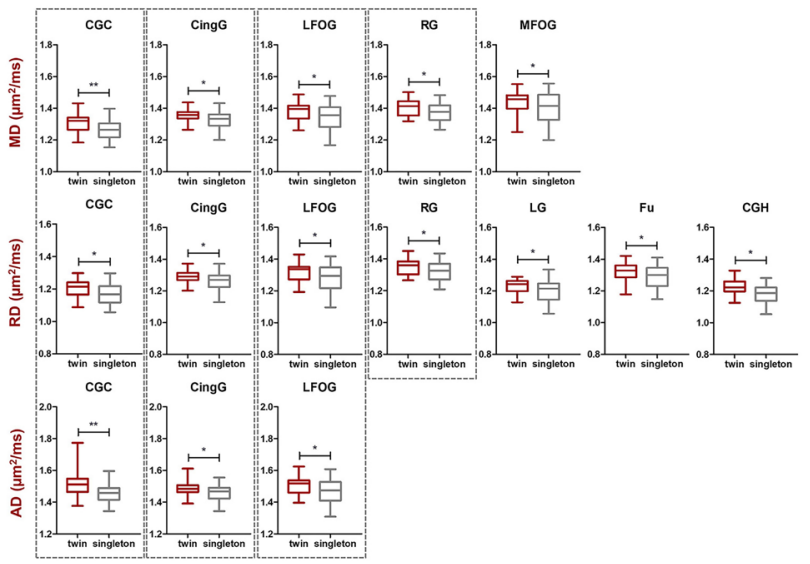

Figure 1. Differences in diffusivity measurements between twin and singleton brains. The MD values in the CGC, CingG, LFOG, RG, and MFOG were higher in the twin group compared with the singletons after multiple comparison correction (adjusted $p<0.05$ ). The corresponding $R D$ and $A D$ values were also higher in the twin group

${ }^{*} p<0.05,{ }^{* *} p<0.01$ by ANCOVA tests followed by Bonferroni correction CGC: Cingulum cingular part, CingG: Cingulate gyrus, LFOG: Lateral fronto-orbital gyru, RG: Gyrus rectus, MFOG: Medial fronto-orbital gyrus, LG: Lingual gyrus, Fu: Fusiform gyrus, CGH: Cingulum hippocampal part, ANCOVA: Analysis of covariance, AD: Axial diffusivity, RD: Radial diffusivity, MD: Mean diffusivity calculating the mean MD difference between the groups normalized to the mean MD of the singleton group for each ROI. No significant difference in FA was found between the twin and singleton groups.

\section{Comparison of the structural connectivity}

The degree, transitivity, local efficiency, global efficiency, modularity, characteristic path length, and smallworldness of the tractography-based structural network were calculated. Only the transitivity, which reflected local connectivity (39), was found to be significantly higher in the twin group than that in the singleton group (Figure 3), and the result did not change with cut-off thresholds (weakest

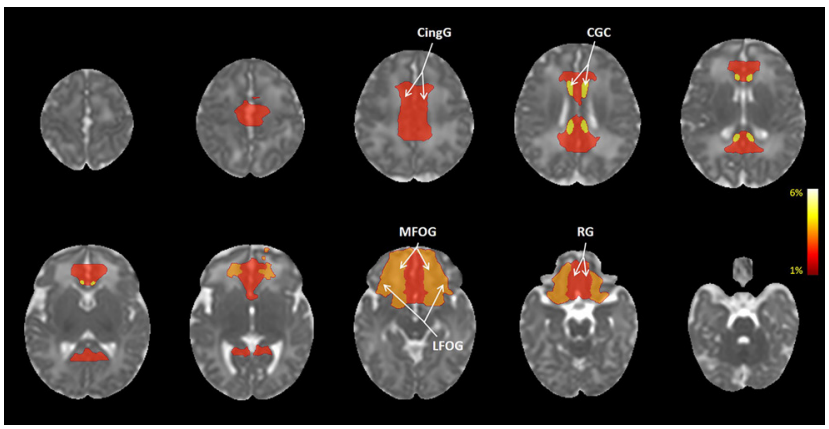

Figure 2. Brain regions showed significantly higher $M D$ in the twin group than in the singleton group. The colors indicate the percentage of group difference by calculating the mean MD difference between the groups normalized to the mean MD of the singleton group for each ROI CGC: Cingulum cingular part, CingG: Cingulate gyrus, LFOG: Lateral fronto-orbital gyru, RG: Gyrus rectus, MFOG: Medial fronto-orbital gyrus, MD: Mean diffusivity, ROI: Region of interest

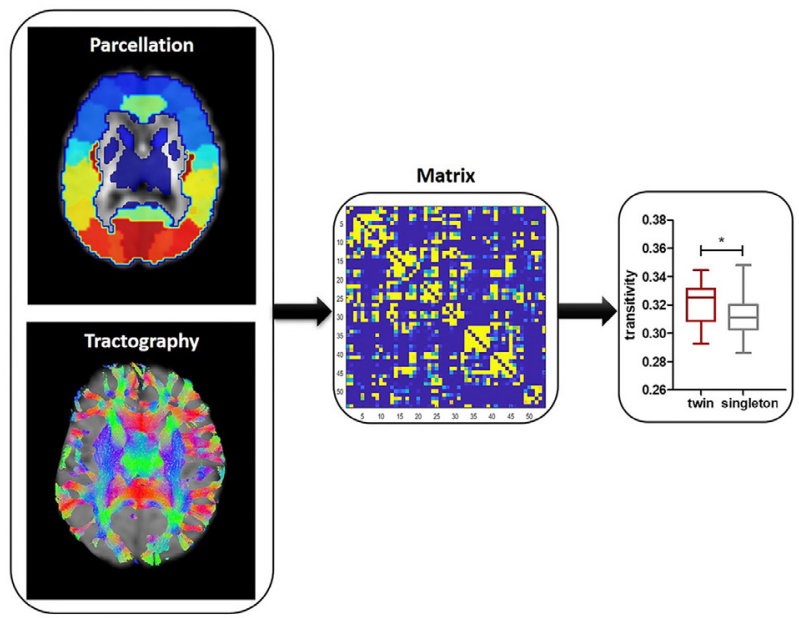

Figure 3. Flow chart of structural network analysis and the statistical result. The DTI data were segmented based on JHU neonatal DTI atlas. Sixty-two ROIs, including cortical GM, deep GM, and subcortical WM, were chosen as the nodes. Tensor-based probabilistic tractography was performed and the fiber accounts between each pair of ROIs were used to obtain the connectivity matrix. Standard network attributes were obtained, and transitivity was found to be significantly higher in the twin group

DTI: Diffusion tensor imaging, ROI: Region of interest 
1-5\% connections) (Supplementary Table III). Transitivity is a classical variant of the traditional clustering coefficient (40), which is thought to be less biased towards the contribution from low-degree vertices.

\section{Comparison of the rs-fMRI results}

As demonstrated in Figure 4, fALFF values in the salience network, which included bilateral insular cortex (Ins) and bilateral cingulate cortex (CingG), and several gyral regions, including the bilateral superior temporal gyrus, bilateral LFOG and bilateral RG, and left postcentral gyrus (POCG_L), were found to be significantly higher in the twin group. Moreover, the fALFF results overlapped with the MD results in several regions, including the CingG, LFOG, and RG (Figure 5). We repeated the analysis with global signal

Supplementary Table III. Comparison of the tractographybased structural network properties between the singleton group and the twin group

\begin{tabular}{|c|c|c|c|c|c|}
\hline Threshold & $1 \%$ & $2 \%$ & $3 \%$ & $4 \%$ & $5 \%$ \\
\hline Degree & 0.067 & 0.051 & 0.056 & 0.083 & 0.091 \\
\hline Transitivity & 0.014 & 0.024 & 0.012 & 0.024 & 0.024 \\
\hline Modularity & 0.196 & 0.166 & 0.141 & 0.134 & 0.140 \\
\hline Character path length & 0.902 & 0.902 & 0.901 & 0.902 & 0.902 \\
\hline Global efficiency & 0.249 & 0.098 & 0.079 & 0.211 & 0.251 \\
\hline Local efficiency & 0.251 & 0.138 & 0.100 & 0.197 & 0.312 \\
\hline Small worldness & 0.075 & 0.158 & 0.132 & 0.047 & 0.200 \\
\hline \multicolumn{6}{|c|}{$\begin{array}{l}\text { Significance levels ( } p \text {-values) for seven network properties were listed under } \\
\text { a range of cut-off thresholds. The cut-off was set to be the weakest 1-5\% } \\
\text { connections }\end{array}$} \\
\hline
\end{tabular}

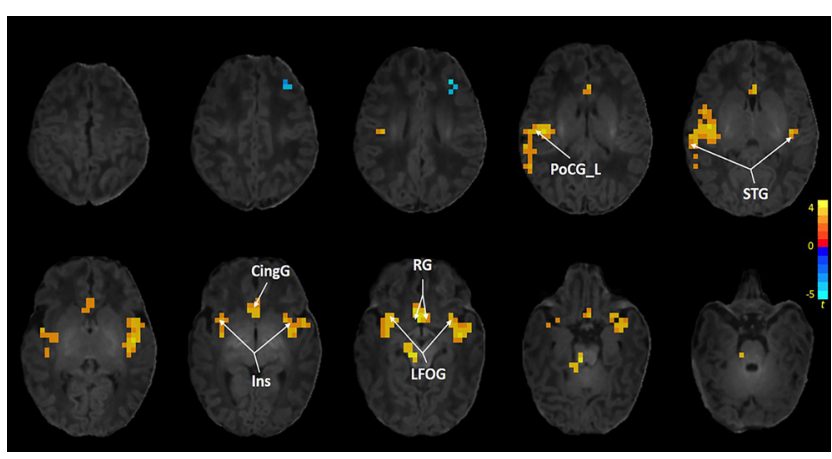

Figure 4. Differences in fALFF between twins and singletons, which were mainly located at the salience network and several fronto-temporal regions. The warm colors indicate higher fALFF in the twins compared with singletons, while the cool colors indicate lower fALFF in the twins compared with singletons. Voxel-based analysis of differences between the groups was performed using Bonferroni correction followed by a threshold-free cluster enhancement method for multiple testing Ins: Insular cortex, CingG: Cingulate cortex, STG: Superior temporal gyrus, LFOG: Lateral fronto-orbital gyrus, RG: Gyrus rectus, POCG_L: Left postcentral gyrus, fALFF: Fractional amplitude of low-frequency fluctuation regression, and the results remained largely unchanged (Supplementary Figure 1). No group difference was found in $A L F F$, and no functional network parameter was found to be different in this study.

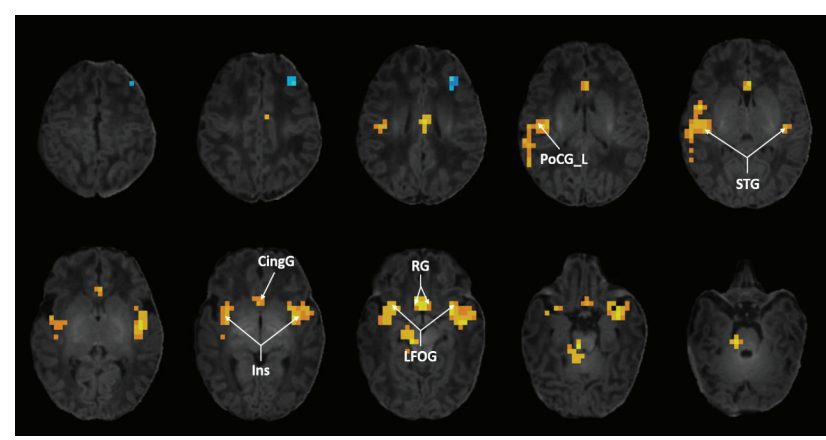

Supplementary Figure 1. Regions that showed significant higher fALFF in the twin group compared with the singleton group after global signal regression. The spatial distribution was similar to that without global signal regression in Figure 5

Ins: Insular cortex, CingG: Cingulate cortex, STC: Superior temporal gyrus, LFOG: Lateral fronto-orbital gyrus, RG: Gyrus rectus, POCG_L: Left postcentral gyrus

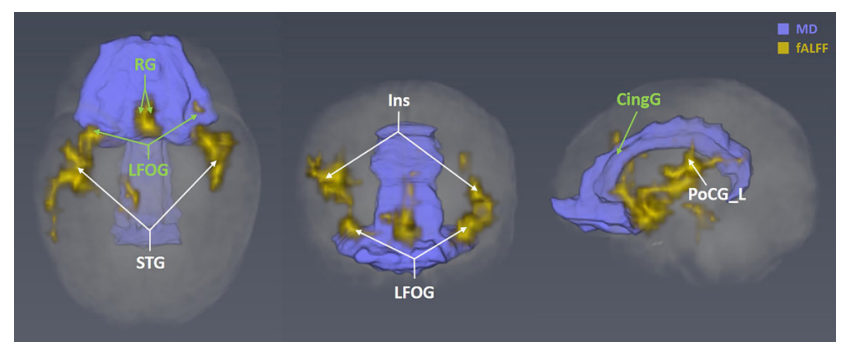

Figure 5. Three-dimensional spatial distribution of the brain regions that show significant group difference in MD (purple) and fALFF (yellow). The fALFF results overlap with the MD results in several regions, including the CingG, LFOG, and RG (green arrow)

Ins: Insular cortex, CingG: Cingulate cortex, STG: Superior temporal gyrus, LFOG: Lateral fronto-orbital gyrus, RG: Gyrus rectus, POCG L: Left postcentral gyrus, fALFF: Fractional amplitude of low-frequency fluctuation

\section{Discussion}

In this study, we performed a comprehensive multimodal MRI study to examine the structural and functional features in twin and singleton brains at term-equivalent age. Our results revealed considerable developmental differences between the two groups compared to previously reported findings, possibly due to the fact this study recruited gestational age-matched twins and singletons whereas previous studies compared preterm-born twins with term-born singletons. Although the gestational age could be included as a covariate, it is difficult to assess how well the effects of the preterm-birth and twin-birth were separated. Therefore, a direct comparison of preterm-born twins and singletons is necessary. 
The volumetric differences were negligible after multiple comparison correction, which was consistent with some of the earlier studies. Knickmeyer et al. (10) compared brain volumes between twins and term-born singletons in the first month of life, and found CSF and frontal white matter volumes were greater in twins than in singletons. Ordaz et al. (11) compared brain volumes between twins and sexmatched unrelated singletons at the pediatric stage, and no significant difference was found. Another morphology study by Hulshoff et al. (9) compared monozygotic twins and dizygotic twins with their siblings in adulthood and found the difference in white matter volume diminished after correction for intracranial volume. Our finding is consistent with the latter two studies but not the first one, which is possibly related to the study population, as well as the use of different brain atlases.

For the DTI measurements, the diffusivities in several cortical gyri, especially the cingulate and front-orbital regions, demonstrated higher values in the twin group than in the singleton group. To the best of our knowledge, only one existing research studied the difference between twins and singletons using diffusion MRI. Sadeghi et al. (12) compared the longitudinal development of white matter between twins and term-born singletons from birth to 2 years of age using a non-linear mixed-effects method, and found the delay parameter of the developmental curve of $A D$ in the anterior limb of the internal capsule and anterior corona radiata was smaller in twins compared to singletons, indicating higher $A D$ in twins during early development. This result is consistent with ours, and their reported regions also showed differences in both FA and MD in our study before multiple comparison correction. However, our data revealed additional regions with higher diffusivities, which is again related to the study population, as well as the use of different brain atlas. Since it is known that the MD value increases with age (41-43), our results indicated a developmental delay in several cortical regions in twins compared with singletons.

For the tractography-based structural connectivity, both groups displayed small-worldness in the wholebrain networks. The transitivity, which indicates local connectivity, was found to be significantly higher in the twins than in singletons. Previous evidence indicated the clustering coefficient of the structural network (similar to transitivity) decreased with brain development from neonate to adult $(44,45)$. However, during perinatal development, some studies found that the preterm-born neonates exhibited an age-dependent increase of clustering coefficient until about term-equivalent age $(46,47)$. These studies indicate the network properties may change in a non-linear pattern during early development, as the brain connectomes experienced ordered strengthening of short-range connectivity followed by growth of long-range connections (48). In the present study, the PMA at scan happened to be the breakpoint of a non-linear trajectory, making it difficult to interpret the developmental difference between twins and singletons. Further longitudinal followup studies may be needed to understand the network difference at term-age.

In the rs-fMRI analyses, fALFF values in the salience network and several fronto-temporal regions were found to be significantly higher in the twin group, while no difference in ALFF was found. The discordance of the two parameters may be due to the artifact generated by head motion. Although sedation was used, neonatal subjects still showed more pronounced motion artifacts than adults, which may not be entirely eliminated by motion correction. The fALFF is a normalized version of ALFF, which is thought to be more robust against physiological artifacts and more sensitive to biological difference $(34,49)$. Few studies have investigated the relationship between low-frequency fluctuation and brain development. Bray (50) studied children aged 7-18 years and found that age did not have a significant effect on global fALFF, but the fALFF in the salience network regions demonstrated an age-related decline. Although no neonatal study of fALFF was found, given the above evidence, our results indicate that preterm-born twins with higher fALFF demonstrated a more active spontaneous neuronal activity, which may be associated with delayed neurological development.

It is worth noting that we found differences both in structural and functional measurements (MD and fALFF) in the prefrontal and CingG (indicated with the green arrow in Figure 5), which are involved in several higherorder cognitive functions (51-53). Although the prefrontal and CingG related cognitive functions are immature at an early age $(48,54)$, these regions are known to be rapidly developing during the perinatal stage (55). The agreement between the structural and functional evidence reinforces the differences between twins and singletons at termequivalent age. These differences in brain development may be associated with the early feeding problems that are more frequent for preterm-born twins, as well as other perinatal-neonatal issues in twins. No difference in the functional network was found, which is slightly different from the result of the structural network. As the structural network is known to develop prior to the functional network $(48,56,57)$, discordant findings between the structural 
and functional networks are within expectation (58-60). Moreover, in the current study, both the preterm twins and singletons showed relatively low levels of the functional network due to the immature functional activity in the neonatal brain as well as the use of sedation, so group difference may be difficult to detect.

The current work possesses several limitations. First, this study lacked term-born neonates as a healthy control group. A number of studies have shown that preterm-birth resulted in delayed brain development compared with term-born individuals in terms of DTI metrics $(61,62)$ and brain volumes $(63,64)$, and therefore, we can readily assume that both the preterm-born twins and singletons have altered DTI and volumetric measurements in comparison to healthy controls. Second, information on interventional operations for the preterm-born neonates was absent in this study, which may play a role in interpreting group differences. Although we have excluded those neonates with acquired brain injury on MRI and those with known perinatal diseases, and the Apgar scores at 5 -mins after birth were relatively normal $(9.83 \pm 0.38$ and $9.74 \pm 0.53$ for twins and singlets, $p=0.46$ ), this evidence cannot guarantee the healthy condition of the study subjects. Third, we did not investigate the correlation between clinical assessments and MRI in this study. In fact, we followed some of the subjects and performed the Bayley tests at 12 months of age, and no behavioral difference was found between groups. The high drop-out rate and insufficient number of subjects ( 6 twins and 20 singletons) in this study were not appropriate for statistical analysis. In addition, the number of twins was limited compared with singletons, and therefore, we performed a permutation test for the ANCOVA analysis to overcome imbalanced data in the present study (37). Finally, chloral hydrate was used in this study for sedation. Although sedation is considered safe and is frequently used in infants to reduce head motion in fMRI (65), a previous study has shown that sedation can induce a reduction in brain activity in infants (66). This could have had an impact on the rs-fMRI results. Future studies should consider and mitigate for this factor to improve the reliability of their results. Nevertheless, this was the first attempt to systematically evaluate both the structural and functional differences between twins and gestationalage matched singletons, and the DTI and rs-fMRI results collectively implied a developmental delay in twins at termequivalent age.

\section{Conclusion}

In summary, the multi-modal MRI approach provided comprehensive information about the developmental differences between twins and singletons, compared with the previous single modal approach. Our results demonstrated that the preterm-born twins had higher MD in several cortical regions compared with gestational-age matched singletons at term-equivalent age. In addition, transitivity of tractography-based structural network and fALFF in the salient network were found to be higher in the twin group. These structural and functional differences collectively indicated that preterm-born twins may have delayed brain development compared with gestational age-matched singletons at term-equivalent age, which may be related to perinatal- neonatal problems. Therefore, MRI findings from twin studies on brain development should only be cautiously generalized to singletons, especially at term-equivalent age.

\section{Acknowledgment}

This work was supported by the Ministry of Science and Technology of the People's Republic of China (2018YFE0114600), the National Natural Science Foundation of China (61801424, 81971606, 91859201, 61801421, and 81971605), the Fundamental Research Funds for the Central Universities of China (2019QNA5024 and 2019FZJD005), and the Scientific Research Fund of Zhejiang Provincial Education Department (Y201431325).

\section{Ethics}

Ethics Committee Approval: Ethical approval was obtained from the Institutional Review Board at the Children's Hospital of Zhejiang University School of Medicine (2019-11-13).

Informed Consent: Written informed consent was provided by the parents.

Peer-review: Externally and internally peer-reviewed.

\section{Authorship Contributions}

Concept: D.W., Design: D.W., Data Collection or Processing: H.Z., Y.Y., T.L., X.S., F.T., Analysis or Interpretation: T.L., Literature Search: W.Z., Z.Z., Y.Z., Writing: T.L.

Conflict of Interest: No conflict of interest was declared by the authors.

Financial Disclosure: The authors declared that this study received no financial support.

\section{References}

1. Baare WF, Hulshoff Pol HE, Boomsma DI, et al. Quantitative genetic modeling of variation in human brain morphology. Cereb Cortex 2001; 11:816-24.

2. Brun CC, Lepore $N$, Pennec $X$, et al. Mapping the regional influence of genetics on brain structure variability--a tensorbased morphometry study. Neuroimage 2009; 48:37-49. 
3. Hulshoff Pol HE, Schnack HG, Posthuma D, et al. Genetic contributions to human brain morphology and intelligence. I Neurosci 2006; 26:10235-42.

4. Peper JS, Schnack HG, Brouwer RM, et al. Heritability of regional and global brain structure at the onset of puberty: a magnetic resonance imaging study in 9-year-old twin pairs. Hum Brain Mapp 2009; 30:2184-96.

5. Maggioni E, Squarcina L, Dusi N, Diwadkar VA, Brambilla P. Twin MRI studies on genetic and environmental determinants of brain morphology and function in the early lifespan. Neurosci Biobehav Rev 2020; 109:139-49.

6. Bryan SM, Hindmarsh PC. Normal and abnormal fetal growth. Horm Res 2006; 65(Suppl 3):19-27.

7. Ounsted $M$, Ounsted C. Maternal regulation of intra-uterine growth. Nature 1966; 212:995-7.

8. Hall JG. Twinning. Lancet 2003; 362:735-43.

9. Hulshoff Pol HE, Posthuma D, Baare WF, et al. Twin-singleton differences in brain structure using structural equation modelling. Brain 2002; 125:384-90.

10. Knickmeyer RC, Kang C, Woolson S, et al. Twin-singleton differences in neonatal brain structure. Twin Res Hum Genet 2011; 14:268-76.

11. Ordaz S), Lenroot RK, Wallace $\mathrm{GL}$, et al. Are there differences in brain morphometry between twins and unrelated singletons? A pediatric MRI study. Genes Brain Behav 2010; 9:288-95.

12. Sadeghi N, Gilmore JH, Gerig C. Twin-singleton developmental study of brain white matter anatomy. Hum Brain Mapp 2017; 38:1009-24.

13. Ombelet $W$, Martens $G$, De Sutter $P$, et al. Perinatal outcome of 12,021 singleton and 3108 twin births after non-IVF-assisted reproduction: a cohort study. Hum Reprod 2006; 21:1025-32.

14. Muhlhausler BS, Hancock SN, Bloomfield FH, Harding R. Are twins growth restricted? Pediatr Res 2011; 70:117-22.

15. Mori S, Wu D, Ceritoglu C, et al. MRICloud: delivering highthroughput mri neuroinformatics as cloud-based software as a service. Comput Sci Eng 2016; 18:21-35.

16. Otsuka Y, Chang L, Kawasaki Y, et al. A multi-atlas label fusion tool for neonatal brain mri parcellation and quantification. I Neuroimaging 2019; 29:431-9.

17. Woods RP, Grafton ST, Holmes C), Cherry SR, Mazziotta IC. Automated image registration: I. General methods and intrasubject, intramodality validation. / Comput Assist Tomogr 1998; 22:139-52.

18. Veraart J, Novikov DS, Christiaens D, Ades-Aron B, Sijbers I, Fieremans E. Denoising of diffusion MRI using random matrix theory. Neuroimage 2016; 142:394-406.

19. Kellner E, Dhital B, Kiselev VG, Reisert M. Gibbs-ringing artifact removal based on local subvoxel-shifts. Magn Reson Med 2016 76:1574-81.

20. Andersson JL, Skare S, Ashburner J. How to correct susceptibility distortions in spin-echo echo-planar images: application to diffusion tensor imaging. Neu roimage 2003; 20:870-88.

21. Tustison NJ, Avants BB, Cook PA, et al. N4ITK: improved N3 bias correction. IEEE Trans Med Imaging 2010; 29:1310-20.
22. Oishi K, Mori S, Donohue PK, et al. Multi-contrast human neonatal brain atlas: application to normal neonate development analysis. Neuroimage 2011; 56:8-20.

23. Christensen GE, Rabbitt RD, Miller MI. Deformable templates using large deformation kinematics. IEEE Trans Image Process 1996; 5:1435-47

24. Miller MI, Christensen GE, Amit Y, Grenander U. Mathematical textbook of deformable neuroanatomies. Proc Natl Acad Sci U S A 1993; 90:11944-8.

25. Djamanakova A, Faria AV, Hsu l, et al. Diffeomorphic brain mapping based on T1-weighted images: improvement of registration accuracy by multichannel mapping. I Magn Reson Imaging 2013; 37:76-84

26. Jones DK. Tractography gone wild: probabilistic fibre tracking using the wild bootstrap with diffusion tensor MRI. IEEE Trans Med Imaging 2008; 27:1268-74.

27. Bonilha L, Gleichgerrcht E, Fridriksson I, et al. Reproducibility of the structural brain connectome derived from diffusion tensor imaging. PLoS One 2015; 10:e0135247. doi: 10.1371/journal. pone.0135247

28. Buchanan $C R$, Pernet $C R$, Gorgolewski KJ, Storkey AJ, Bastin ME. Test-retest reliability of structural brain networks from diffusion MRI. Neuroimage 2014; 86:231-43.

29. Yap PT, Fan Y, Chen Y, Gilmore JH, Lin W, Shen D. Development trends of white matter connectivity in the first years of life. PLoS One 2011; 6:e24678. doi: 10.1371/journal.pone.0024678.

30. Sotiropoulos SN, Zalesky A. Building connectomes using diffusion MRI: why, how and but. NMR Biomed 2019; 32:e3752. doi: $10.1002 / \mathrm{nbm} .3752$.

31. Rubinov M, Sporns O. Complex network measures of brain connectivity: uses and interpretations. Neuroimage 2010; 52:1059-69.

32. 32. Yan CG, Wang XD, Zuo XN, Zang YF. DPABI: data processing \& analysis for (resting-state) brain imaging. Neuroinformatics 2016; 14:339-51

33. Jenkinson $M$, Bannister $P$, Brady $M$, Smith $S$. Improved optimization for the robust and accurate linear registration and motion correction of brain images. Neuroimage 2002; 17:825-41.

34. Zuo XN, Di Martino A, Kelly $C$, et al. The oscillating brain: complex and reliable. Neuroimage 2010; 49:1432-1445.

35. Mukaka MM. Statistics corner: a guide to appropriate use of correlation coefficient in medical research. Malawi Med I 2012; 24:69-71.

36. Team RC. A language and environment for statistical computing. 2013 Available from: http://www.R-project.org/

37. Wheeler REJTCRAN 2010 Permutation tests for linear models in R. 1. Available from: https://cran.r-project.org/web/packages/ ImPerm/vignettes/lmPerm.pdf

38. Smith SM, Nichols TE. Threshold-free cluster enhancement: addressing problems of smoothing, threshold dependence and localisation in cluster inference. Neuroimage 2009; 44:83-98.

39. Newman ME). 2003 The structure and function of complex networks. Siam Rev 2003; 45:167-256.

40. Watts DJ, Strogatz SH. 1998 Collective dynamics of 'smallworld' networks. Nature 1998; 393:440-2. 
41. Dubois J, Dehaene-Lambertz G, et al. Asynchrony of the early maturation of white matter bundles in healthy infants: Quantitative landmarks revealed noninvasively by diffusion tensor imaging. Hum Brain Mapp 2008; 29:14-27.

42. Dubois J, Hertz-Pannier L, Dehaene-Lambertz G, Cointepas Y, Le Bihan D. Assessment of the early organization and maturation of infants' cerebral white matter fiber bundles: a feasibility study using quantitative diffusion tensor imaging and tractography. Neuroimage 2006; 30:1121-32.

43. Wu D, Chang LD, Akazawa K, et al. Mapping the critical gestational age at birth that alters brain development in preterm-born infants using multi-modal MRI. Neuroimage 2017; 149:33-43.

44. Tymofiyeva O, Hess CP, Ziv E, et al. A DTI-based template-free cortical connectome study of brain maturation. PLoS One 2013;8:e63310. doi: 10.1371/journal.pone.0063310.

45. Hagmann P, Sporns O, Madan N, et al. White matter maturation reshapes structural connectivity in the late developing human brain. Proceedings of the National Academy of Sciences of the United States of America 2010; 107:19067-72.

46. Brown C), Miller SP, Booth BG, et al. Structural network analysis of brain development in young preterm neonates. Neuroimage 2014; 101:667-680.

47. van den Heuvel MP, Kersbergen KJ, de Reus MA, et al. The neonatal connectome during preterm brain development. Cerebral Cortex 2015; 25:3000-13.

48. Cao M, Huang $\mathrm{H}$, He Y. Developmental connectomics from infancy through early childhood. Trends Neurosci 2017; 40:494506.

49. Zou $\mathrm{QH}$, Zhu $\mathrm{CZ}$, Yang Y, et al. An improved approach to detection of amplitude of low-frequency fluctuation (ALFF) for resting-state fMRI: fractional ALFF. I Neurosci Methods 2008; 172:137-41.

50. Bray S. Age-associated patterns in gray matter volume, cerebral perfusion and bold oscillations in children and adolescents. Human Brain Mapp 2017; 38:2398-407.

51. Rudebeck PH, Behrens TE, Kennerley SW, et al. Frontal cortex subregions play distinct roles in choices between actions and Stimuli. / Neurosci 2008; 28:13775-85.

52. Rushworth MF, Behrens Choice, uncertainty and value in prefrontal and cingulate cortex. Nat Neurosci 2008; 11:389-97.

53. Rushworth MF, Behrens TE, Rudebeck PH, Walton ME. Contrasting roles for cingulate and orbitofrontal cortex in decisions and social behaviour. Trends Cogn Sci 2007; 11:168-76.
54. Happaney K, Zelazo PD, Stuss DT. Development of orbitofrontal function: current themes and future directions. Brain Cogn 2004; 55:1-10

55. Ouyang $M$, Jeon $T$, Sotias $A$, et al. Differential cortical microstructural maturation in the preterm human brain with diffusion kurtosis and tensor imaging. Proc Natl Acad Sci U S A 2019; 116:4681-8.

56. Park HJ, Friston K. Structural and functional brain networks: from connections to cognition. Science 2013; 342:1238411. doi: $10.1126 /$ science. 1238411

57. Wang ZI, Dai ZI, Gong GL, Zhou CS, He Y. Understanding structural-functional relationships in the human brain: a largescale network perspective. Neuroscientist 2015; 21:290-305.

58. Hagmann $P$, Cammoun L, Gigandet $X$, et al. Mapping the structural core of human cerebral cortex. PLoS Biol 2008; 6:e159. doi: 10.1371/journal.pbio.0060159.

59. Honey C), Kotter R, Breakspear M, Sporns O. Network structure of cerebral cortex shapes functional connectivity on multiple time scales. Proc Natl Acad Sci U S A 2007; 104:10240-5.

60. Honey Cl, Sporns O, Cammoun L, et al. Predicting human restingstate functional connectivity from structural connectivity. Proc Natl Acad Sci U S A 2009; 106:2035-40.

61. Eikenes L, Lohaugen CC, Brubakk AM, Skranes J, Haberg AK. Young adults born preterm with very low birth weight demonstrate widespread white matter alterations on brain DTI. Neuroimage 2011; 54:1774-85.

62. Vangberg TR, Skranes I, Dale AM, Martinussen M, Brubakk AM Haraldseth $O$. Changes in white matter diffusion anisotropy in adolescents born prematurely. Neuroimage 2006; 32:1538-48.

63. Nosarti C, Al-Asady MH, Frangou S, Stewart AL, Rifkin L, Murray RMJB. Adolescents who were born very preterm have decreased brain volumes. Brain 2002; 125:1616-23.

64. Soria-Pastor S, Padilla N, Zubiaurre-Elorza L, et al. Decreased regional brain volume and cognitive impairment in preterm children at low risk. Pediatrics 2009; 124:e1161-70. doi: 10.1542/ peds.2009-0244.

65. Finnemore $A$, Toulmin $H$, Merchant $N$, et al. Chloral hydrate sedation for magnetic resonance imaging in newborn infants. Pediatr Anesth 2014; 24:190-5.

66. Williams G, Fabrizi L, Meek J, et al. Functional magnetic resonance imaging can be used to explore tactile and nociceptive processing in the infant brain. Acta Paediatr 2015; 104:158-66. 